\title{
Obstetric outcomes of women referred for delivery to a tertiary hospital in northern Tanzania- a descriptive retrospective study based on a hospital birth registry
}

Carolyn Lissu

Faculty of Nursing

Helena Volgsten ( $\square$ helena.volgsten@kbh.uu.se )

Dept Womens and Childrens Health https://orcid.org/0000-0003-0180-0280

Festu Mazuguni

KCMUC

Eusebious Maro

dept of obstetrics and gynecology

Research

Keywords: maternal mortality, obstetric outcome, referral system, Tanzania

Posted Date: February 20th, 2020

DOI: https://doi.org/10.21203/rs.2.24072/v1

License: (c) (1) This work is licensed under a Creative Commons Attribution 4.0 International License.

Read Full License 


\section{Abstract}

Background: Maternal mortality remains a great challenge in a low-income country like Tanzania, despite global and national efforts to improve women's reproductive health. Timeliness and appropriateness of referral from a lower- to higher-level health facility comprise an important factor for the obstetrics outcome for pregnant women. This study aimed to determine the obstetric outcomes, such as maternal deaths, of women referred to KCMC, a tertiary hospital in northern Tanzania.

Methods: A descriptive retrospective study based on a hospital birth registry was conducted, using consecutive stored data on pregnant women referred while in labor and managed at the KCMC tertiary hospital in northern Tanzania between the years 2000 and 2015. All referred pregnant women whose labor status information was missing during admission were excluded from the study.

Results: During the study period, a total of 53662 deliveries were managed at KCMC. Among these, 6066 women were referred from lower health facilities, with $4193(69.2 \%)$ of them being referred while in labor. The main reason for referral was poor progress of labor (31.0\%), followed by prolonged labor $(27.1 \%)$ and obstructed labor (19.5\%). For 1859 (44.6\%) women, delivery was by caesarean section. A total of 292 maternal deaths occurred between 2000 and 2015. Of these, almost a quarter (22.6\%) occurred in women referred from other health facilities while in labor.

Conclusions: Most of the maternal complications during labor and delivery were prevalent among women referred from lower health facilities. This underscores the need to strengthen lower health facilities' ability to detect complications in timely manner and provide effective emergency obstetric care, as well as to refer women to higher-level facility.

\section{Plain English Summary}

High maternal deaths in low-income settings are attributed to poor emergency obstetric care. This study investigated maternal outcomes for women referred during labor to a tertiary-level hospital in northern Tanzania. Maternal complications were prevalent, with almost a quarter ending in maternal death. There is a need to strengthen lower health facilities in their ability to detect complications and provide effective emergency obstetric care, as well as timely referral to higher-level facility. Community empowerment to avoid initial delays cannot be overemphasized in a low-income country like Tanzania.

\section{Background}

Maternal mortality during pregnancy and childbirth remains a great challenge in many low-income African countries like Tanzania, despite global and national efforts to improve women's reproductive health. Tanzania has the fourth highest number of maternal deaths in Sub-Saharan Africa and the sixth highest in the world [1], with a maternal mortality ratio (MMR) of 556/100.000 live births in 2015 [2]. Obstetric emergencies are the leading cause of maternal, fetal, and neonatal mortality, particularly in low- 
income countries [3]. This high number of maternal deaths is even threatening the new target of Sustainable Development Goal (SDG) 3.1, to be achieved by 2030 [4].

Tanzania's healthcare system is a pyramid structure, providing its referral services from primary-level healthcare to secondary (district \& regional) and tertiary (central/consultant) hospitals or specialized hospitals [5]. A woman reporting labor pain at a primary healthcare facility is usually admitted for observation of labor progress. In the case of complications or any risk factors, a doctor is informed and the matter is discussed by the doctors and midwives. If the facility lacks the ability to handle complications, the woman is transferred to a higher-level facility (secondary or tertiary hospital) [6].

The timely identification of danger signs in pregnant women before developing complications, as well as the identification of these patients and their prompt referral to a center that is well equipped to tackle such cases, may improve the maternal outcome [7]. In the absence of interventions and a proper referral system, there is usually high maternal morbidity and mortality. Referral due to obstetric complications offers women some degree of care at every level of the healthcare system [8].

Most of the women referred for delivery in tertiary hospitals are in labor, and hence need to be admitted. Cross-sectional studies of referred obstetric emergencies show that $42-68 \%$ of these were admitted with labor pain [8-9]. Direct causes of maternal death, such as hemorrhage, infection, high blood pressure, unsafe abortion, and obstructed labor, comprised the majority of these referrals and admissions. These direct causes are currently still the reasons for the referral of pregnant women as well as for poor obstetric outcomes [5].

A number of interventions have been put in place by the government of Tanzania, such as training healthcare providers in Basic Emergency Obstetric Care and Comprehensive Emergency Obstetric Care, and ensuring a constant supply of essential equipment, aiming to reduce maternal and neonatal morbidity and mortality. These efforts are aimed at giving healthcare providers at each level the ability to handle and reduce the obstetric complications that cause increased obstetric referrals, as well as decreasing maternal and fetal morbidity and mortality [10-12].

Despite the efforts described, there are still instances of suboptimal care in health facilities, especially in rural Tanzania [13]. The timeliness and appropriateness of referral form an important factor in the fate of pregnant women and their obstetric outcomes. However, this still faces practical challenges due to many circumstances. The three-delay model identifies three groups of factors that contribute to difficulties in women accessing care and poor obstetric outcomes: Delay in the decision to seek care, Delay in reaching care; and Delay in receiving adequate healthcare. Together, these expose laboring women to unnecessary medical and surgical interventions [14].

Being a country with a high MMR, Tanzania needs to update its information on the proportion of women referred to tertiary healthcare, their demographic and obstetric characteristics and outcomes. Hence, this study aimed to determine the obstetric outcomes, such as maternal deaths, of women referred to KCMC, a tertiary hospital in northern Tanzania. 


\section{Materials And Methods \\ Study design}

A descriptive retrospective study based on a hospital birth registry, including consecutive stored data on pregnant women in labor referred to and managed at the Kilimanjaro Christian Medical Centre (KCMC) tertiary hospital in northern Tanzania between the years 2000 and 2015.

\section{Study setting}

The study was conducted at the KCMC hospital, located in Moshi Municipality in the Kilimanjaro region in northern Tanzania. KCMC is a zonal consultant hospital that provides tertiary (Level 3) medical services for the regions within Tanzania's Northern Zone, as well as from neighboring districts in Kenya close to the border. The hospital serves a population of more than 15 million and has a bed capacity of 450; however, its daily patient census ranges from 500 to 550 [15]. The average annual birth rate at KCMC is about 4000 births. Its labor ward receives normal deliveries, as well as high-risk women with obstetric complications at various stages of pregnancy, labor or delivery, referred from health facilities in the Northern Zone. The labor ward has four beds as well as a clean corridor, which sometimes serves as additional patient space. Its obstetrics and gynecology department has seven full-time obstetricians, who are always available to attend to these referrals.

\section{Data collection}

The Medical Birth Registry is a tool that contains information on all births at KCMC and is intended to serve clinical, administrative, and research purposes. The rationale for this registry is to collect data in order to develop and organize maternity care, obstetrical services, and neonatal care. The registry was begun in July 2000 , with each birth being recorded in a separate database. The information is obtained through daily personal interviews with each mother within 24 hours after delivery.

A standardized data extraction sheet was used to collect all relevant information in the Medical Birth Registry pertaining to the study variables [16]. All referred pregnant women noted to be missing information in the registry upon admission regarding their labor status were excluded. Variables of interest were socio-demographic and obstetric characteristics, including maternal outcomes such as mode of delivery, complications (postpartum hemorrhage, pre-eclampsia, eclampsia), trauma (episiotomy, perineal tear), and maternal death.

\section{Data processing and analysis}

Data were checked for completeness and accuracy prior to analysis. All data processing and analysis was performed using the Statistical Package for Social Sciences (SPSS) version 23. Data on categorical variables were summarized using frequencies and percentages, and using mean and standard deviation (SD) for continuous variables.

\section{Ethical consideration}


Ethical approval was obtained from the Kilimanjaro Christian Medical University College Research and Ethics Committee, registration No. 1008. Permission to access the database was received from the KCMC hospital administration.

\section{Results}

During the study period, a total of 53662 deliveries were managed at KCMC. Among these, a total of 6066 women were referred from lower health facilities, of whom 4193 (69.2\%) were referred while in labor and were included in the study, as presented in Fig. 1.

The mean age was $25.7 \pm 6.4$, and most $(73.4 \%)$ of the women had a primary education. The majority of the women in labor (75.4\%) were from rural areas, although they were equally referred from district and regional hospitals; socio-demographic characteristics are presented in Table 1. More than half $(54.9 \%)$ of the women were referred as primigravida, and the majority $(71.9 \%)$ were at term gestation. Moreover, the leading reason for referral was poor progress of labor (31.0\%), followed by prolonged labor and obstructed labor at $27.1 \%$ and $19.5 \%$ respectively; obstetric characteristics are presented in Table 2 . 
Table 1

Socio-demographic characteristics of women referred for delivery at KCMC from 2000 to $2015(n=4193)$

\begin{tabular}{|ll|}
\hline Characteristics & $\mathbf{n}(\%)$ \\
\hline Age (years) $\dagger$ & $25.74(6.49)$ \\
\hline Maternal age* & \\
\hline$<20$ & $757(18.06)$ \\
\hline $20-35$ & $2939(70.13)$ \\
\hline$>35$ & $495(11.81)$ \\
\hline Marital status* & \\
\hline Married & $3532(84.46)$ \\
\hline Not Married & $650(15.54)$ \\
\hline Educational Status* & \\
\hline None & $183(4.37)$ \\
\hline Primary & $3072(73.42)$ \\
\hline Secondary & $442(10.56)$ \\
\hline Higher & $487(11.64)$ \\
\hline Religion* & $17612(38.56)$ \\
\hline Catholic & $1163(27.86)$ \\
\hline Protestants & $1881(45.06)$ \\
\hline Muslim & $113(26.67)$ \\
\hline Others & $17.41)$ \\
\hline Mothers tribe* & \\
\hline Chagga & \\
\hline Pare & \\
\hline Masai & \\
\hline Others & \\
\hline Residence & \\
\hline
\end{tabular}




\begin{tabular}{|ll|}
\hline Characteristics & $\mathbf{n}(\%)$ \\
\hline Rural & $3161(75.39)$ \\
\hline Urban & $1032(24.61)$ \\
\hline Referred from* & \\
\hline Regional & $2079(49.67)$ \\
\hline District & $2090(49.93)$ \\
\hline Unknown & $17(0.41)$ \\
\hline tMean (SD), *Missing values \\
\hline
\end{tabular}


Table 2

Obstetric characteristics of women referred for delivery to KCMC from 2000 to $2015(n=4193)$

\begin{tabular}{|ll|}
\hline Characteristics & $\mathbf{n}(\%)$ \\
\hline Parity & $2303(54.90)$ \\
\hline Primipara & $1512(36.05)$ \\
\hline Multipara & $379(9.04)$ \\
\hline Grand multipara & \\
\hline Gestational age (Weeks)* & $24(0.64)$ \\
\hline Extreme Preterm (<28) & $100(2.66)$ \\
\hline Very Preterm (>28-32) & $485(12.91)$ \\
\hline Moderate Preterm(>32-37) & $2704(71.99)$ \\
\hline Term (>37-42) & $443(11.79)$ \\
\hline Post term >42 & \\
\hline Labor Induction* & $865(20.80)$ \\
\hline Yes & $3294(79.20)$ \\
\hline No & \\
\hline BMl* & $324(10.30)$ \\
\hline Normal & $184(2.45)$ \\
\hline Underweight & $551(36.13)$ \\
\hline Overweight & $408(26.75)$ \\
\hline Obese & $115(3.66)$ \\
\hline Reasons for referral* & \\
\hline Prolonged labor & \\
\hline Abruption & \\
\hline Prematurity & \\
\hline Big baby & \\
\hline Early Rapture of membrane & $77.85)$ \\
\hline
\end{tabular}

*Missing values 


\begin{tabular}{|ll|}
\hline Characteristics & $\mathbf{n}(\%)$ \\
\hline Poor progress of labor & $978(31.10)$ \\
\hline Obstructed labor & $613(19.49)$ \\
\hline *Missing values & \\
\hline
\end{tabular}

About 1859 (44.6\%) women had their delivery by caesarean section (CS). Ten percent of these referred women encountered postpartum hemorrhage, while 67 women (1.6\%) experienced bleeding late in their pregnancy with 25 of them having placenta previa and 42 abruptio placenta. About $5.8 \%$ of the referred women had an episiotomy.

During 2000-2015 a total of 292 maternal deaths occurred out of 53662 deliveries. Among 4193 referred women, 66 (1.6\%) maternal deaths were recorded; obstetric outcomes are presented in Table 3. 
Table 3

Maternal outcomes among referred women at

KCMC from 2000 to $2015(n=4193)$

\begin{tabular}{|ll|}
\hline Maternal outcomes & $\mathbf{n}(\%)$ \\
\hline Mode of delivery* & \\
\hline Spontaneous vertex delivery & $2176(52.19)$ \\
\hline Caesarian section & $1859(44.59)$ \\
\hline Assisted vaginal delivery & $134(3.21)$ \\
\hline PPH* & \\
\hline Yes & $277(10.60)$ \\
\hline No & $2337(89.40)$ \\
\hline Eclampsia & \\
\hline Yes & $116(2.77)$ \\
\hline No & $4077(97.23)$ \\
\hline Episiotomy & \\
\hline Yes & $244(5.82)$ \\
\hline No & $3949(9418)$ \\
\hline Placenta Previa & $410(0.23)$ \\
\hline Yes & $25(0.59)$ \\
\hline No & $4168(99.41)$ \\
\hline Abruption placenta & \\
\hline Yes & $4180(99.69)$ \\
\hline No & \\
\hline $3-4$ degree perineal tear & \\
\hline Yes & \\
\hline No & \\
\hline Ruptured Uterus & \\
\hline Yes & \\
\hline
\end{tabular}

*Missing values

Page $10 / 17$ 


\begin{tabular}{|ll|}
\hline Maternal outcomes & $\mathbf{n}(\%)$ \\
\hline No & $4183(99.76)$ \\
\hline Cord prolapsed & \\
Yes & $7(0.16)$ \\
\hline No & $4186(99.84)$ \\
\hline Retained 2nd Twin & \\
\hline Yes & $11(0.26)$ \\
No & $4182(99.74)$ \\
\hline Maternal deaths & \\
\hline Yes & $66(1.57)$ \\
\hline No & $4127(98.43)$ \\
\hline *Missing values & \\
\hline
\end{tabular}

\section{Discussion}

This study found the proportion of pregnant women referred while in labor to be $69 \%$, and the majority of them were primigravida. The main reason for referral was poor progress of labor, and among these women almost half had their delivery by caesarean section. A total of 292 maternal deaths occurred between the years 2000 and 2015. Of these maternal deaths, almost a quarter occurred in women referred from other health facilities while in labor.

The proportion of referred women found in this study is comparable to findings in other studies but in contrast to another, which reported a higher rate (87\%) of referred patients in labor $[9,17-19]$. However, the proportion is higher than in other studies, reporting $60.3 \%$ and $42.2 \%$, respectively $[8,19]$. The possible reasons for these differences in proportion might include the study design as well as study duration and sample size. Another possible explanation could be the difference in size between the health facilities. The present study was conducted at a zonal tertiary hospital serving a large population of more than 15 million.

Referred women in this study, were equally referred from district and regional hospitals (secondary-level health facilities). This similarity could be due to the local referral system within the region that allows patients and their relatives to influence the referring channel. Other explanations could be the severity of the condition of the referred patient or the nearness of the referring health facility to KCMC. Further, more than half of women in labor in northern Tanzania have their delivery at the hospital by a skilled attendant and can access referral to secondary- and tertiary-level of healthcare in the event of a complication [5]. 
The Caesarean section (CS) rate of almost $45.0 \%$ observed in this study is higher than the WHO recommendations [5], which stress that CS rates above $10 \%$ are not associated with a decrease in maternal mortality rates [5]. Similar studies have also reported higher rates of CS among obstetric referrals $[9,18]$. The high rate may be due to prolonged duration of labor at the previous health facility, leading to a compromised maternal-fetal outcome. Nevertheless, the complications experienced by women during labor at any hospital depends upon a number of factors, such as type of obstetric complications, competency of the midwives/doctors, and distance from the health facility in the catchment area to the referred hospital. Other general contributing factors include the socio-economic status and literacy of the parturient, the frequency and quality of prenatal care, and timely referral by the facility [9].

Among the obstetric complications in the referred women, postpartum hemorrhage (PPH) was the most common, reported in $10.6 \%$ of cases. Similar findings have been reported in studies from Uganda and Tanzania, which reported PPH in $9.0 \%$ and $11.9 \%$, respectively [20-21]. However, this is lower than reported in another study [9], which found PPH in $40.0 \%$ of cases. This result is in contrast to the present study, which could be associated with an early anticipation of PPH in these complicated referred cases and preparation in order to prevent it. The use of uterotonic agents and the availability of experienced staff in the application of active management of the third stage of labor is recommended for preventing PPH. Thus, delivery by skilled attendants in hospital should be highly promoted [22]. Even though there have been improvements in management, PPH remains a significant contributor to maternal morbidity and mortality in both developed and developing countries, although the exact frequency of PPH is difficult to determine [23].

An episiotomy rate of $5.8 \%$ of the referred women is lower than the $39.3 \%$ reported in Nigeria [24]. The lower rate found in this study might be explained by the successful efforts of the obstetrics and gynecology department at KCMC that led to the attainment of the recommended WHO target of reducing the episiotomy rate to $10 \%$ [22]. It is likely that midwives are overly supportive when conducting deliveries for the referred women, in the sense that their threshold for performing an episiotomy is higher despite the complicated nature of the referred cases. Constant attendance by a midwife offering continuous care at all stages of labor could be an alternative explanation for the lower rate of episiotomy [25].

A total of 292 maternal deaths occurred in this study, following 15 years of review. This MMR (292/53662) equals 544/100.000 live births in a year, as the MMR in Tanzania in 2015 [2]. Furthermore, of the maternal deaths in this study, almost a quarter (22.6\%) occurred in women referred from other health facilities while already in labor. The proportion of maternal deaths among referred women was also reflected in a previous study at KCMC by Maro et al. [26], who reported an even higher rate of maternal deaths among women referred from other health facilities. A delay in the decision to refer women with complications to an appropriate level of care is one possible explanation for these maternal deaths among parturient. However, a weakened healthcare system at all levels of facilities could be the reason for preventable maternal deaths in low-resource settings, where there is poor reproductive healthcare, including a lack of access to attentive antenatal care, effective referrals, and supervised 
childbirth [27]. Efforts should be made to improve the management of obstetric emergencies at different levels of health facilities, as well as in the communities where most of the women experiencing these deaths came from. Empowering the family and community level by building capacity and clear systems is important for facilitating the referral of pregnant women in need of advanced care.

A limitation of this study is that missing information on study participants from the Medical Birth Registry may have led to their elimination from analysis. However, as the registry is filled in on a daily basis and thus within 24 hours after delivery, most of the information needed was captured accurately and completely, which reduces the risk of recall and misclassification bias. Another limitation is that this is a hospital-based study, and there may be a lack of generalizability. However, being one of the four zonal referral centers, KCMC provided a better study area for understanding the functionality of the referral system in Tanzania, as it receives cases from all levels of healthcare. A strength is that this study is one among few conducted in Tanzania to report the obstetric outcomes of women referred to a tertiary hospital in northern Tanzania for delivery. Moreover, the study was based on authorized birth registry data covering a period of over 15 years, which provided a long enough period to study the outcomes and referral system in the region and the country at large.

\section{Conclusions}

Emergency obstetric care is still a challenge, especially in lower-level health facilities in Tanzania. Most maternal complications occurring during labor and delivery are more prevalent among referred women from the lower health facilities. This underscores the need to strengthen lower health facilities in the ability to quickly detect complications and provide effective emergency obstetric care, as well as timely referral to higher-level facility. However, community empowerment to avoid initial delays cannot be overemphasized in the efforts towards accelerating the attainment of the SDG in a low-income country like Tanzania.

\section{Abbreviations}

CS - Caesarean section, MMR - Maternal mortality ratio, PPH

Postpartum hemorrhage

\section{Declarations}

\section{Ethics approval and consent to participate}

Ethical approval was obtained from the Kilimanjaro Christian Medical University College Research and Ethics Committee, registration No. 1008. Permission to access the database was received from the KCMC hospital administration. 


\section{Consent for publication}

Not applicable

\section{Availability of data and materials}

Data sharing not applicable

\section{Competing interests}

No conflicts of interest declared by the authors

\section{Funding}

Not applicable

\section{Authors' contributions}

CAL and EM contributed to the design of the study. CAL and FM performed the data analysis and interpretation of data for the manuscript; EM and HV contributed to drafting the manuscript and revising it critically for important intellectual content. All authors have read the final version of the manuscript, agreed to be accountable for all aspects of the manuscript, and approved the final manuscript for publication.

\section{Acknowledgements}

The authors would like to thank the KCMC Birth Registry staff for their priceless efforts in the data collection process.

\section{References}


[1] Say L. et al. "Global causes of maternal death: a WHO systematic analysis." Lancet. Glob. Heal., vol. 2, no. 6, pp. e323-33, Jun. 2014.

[2] World Bank, International Comparison Program Database. July 30, 2019

[3] Offerhaus PM. et al. "Persisting rise in referrals during labor in primary midwife-led care in The Netherlands," Birth, vol. 40, no. 3, pp. 192-201, 2013.

[4] WHO, “Maternal mortality key facts 2018." p. 1, 2018.

[5] Ministry of Health, Community Development [Tanzania M. M. of H. (MoH) [Zanzibar]; "Tanzania," Demogr. Heal. Surv., vol. 2, p. 207, 2016.

[6] ANNIE KEARNS, Women and Health Initiative \& Maternal Health Task Force TAYLOR HURST, Harvard School of Public Health JACQUELYN CAGLIA, Women and Health Initiative \& Maternal Health Task Force ANA LANGER, “Focused Antenatal Care in Tanzania," COUNTRY-LEVEL Program., 2014.

[7] Goswami D. and Makhija A. "A Study of High Risk Obstetric Referrals to Tertiary Care Hospital in Garhwal , Uttarakhand," vol. 4, no. 10, pp. 2014-2016, 2015.

[8] Thaker M. et al. "Retrospective comparative study of obstetric complications and maternal mortality in registered and unregistered women at tertiary care hospital," NHL Journal of Medical Science, vol. 2, no. 1. pp. 1-8, 2013.

[9] Ambreen A. et al. "Obstetrics Outcome of Cases Referred to Tertiary Care Hospital after Trial of Labour," vol. 18, no. 1, pp. 71-80, 2012.

[10] Singh S. P. et al. "Referrals between public sector health institutions for women with obstetric high risk, complications, or emergencies in India - A systematic review," PLOS ONE, vol. 11, no. 8. 2016.

[11] Shija AE. et al. "Maternal health in fifty years of Tanzania independence: Challenges and opportunities of reducing maternal mortality," Tanzania Journal of Health Research, vol. 13, no. 5 SUPPL.ISS. pp. 1-15, Dec-2011.

[12] Otolorin E. et al. "Essential basic and emergency obstetric and newborn care: From education and training to service delivery and quality of care," Int. J. Gynecol. Obstet., vol. 130, no. S2, pp. S46-S53, 2015.

[13] Pembe A. et al. "Quality of antenatal care in rural Tanzania: counselling on pregnancy danger signs." BMC Pregnancy Childbirth, vol. 10, p. 35, 2010.

[14] Maternity Worldwide, "The Three Delays Model and our Integrated Approach - Maternity Worldwide." 2015.

[15] Procurement and Authority, "the United Republic of Tanzania," p. 11478, 2014. 
[16] Mann S. et al. "Assessing quality obstetrical care: development of standardized measures.," Jt. Comm. J. Qual. Patient Saf., vol. 32, no. 9, pp. 497-505, 2006.

[17] Patel S. et al. "Study of Maternal and Fetal Outcome of Patients Referred In Third Trimester of Pregnancy at Tertiary Care Hospital," vol. 3, no. September 2014, pp. 2449-2452, 2015.

[18] Narsaria K. "A Study of obstetric referrals-one year experience at a tertiary care centre in West Bengal," HECS Int. J. Community Heal. Med. Res., vol. 3, no. 3, pp. 32-36, 2017.

[19] Mustafa L. et al. "Maternal and Fetal Outcome of Obstetric Emergencies in a Tertiary Health Institution in South-Western Nigeria," ISRN Obstet. Gynecol., vol. 2011, pp. 1-4, 2011.

[20] Stanford BYJ. "Post Partum Haemorhage Among Women Delivered At Mbeya Referral Hospital in 2008 .," Dar-es-salaam Med. Students' J., no. September, pp. 19-23, 2010.

[21] Ononge S. et al. "Incidence and risk factors for postpartum hemorrhage in Uganda.," Reprod. Health, vol. 13, p. 38, Apr. 2016.

[22] WHO, "WHO recommendation on episiotomy policy | RHL," 2018. [Online]. Available: https://extranet.who.int/rhl/topics/preconception-pregnancy-childbirth-and-postpartum-care/care-duringchildbirth/care-during-labour-2nd-stage/who-recommendation-episiotomy-policy-0. [Accessed: 18-Oct2018].

[23] Sørbye IK. et al. "Caesarean section among referred and self-referred birthing women: a cohort study from a tertiary hospital, northeastern Tanzania," BMC Pregnancy Childbirth, vol. 11, no. 1, p. 55, Dec. 2011.

[24] Izuka E. et al. "Prevalence and predictors of episiotomy among women at first birth in enugu, South-East Nigeria.," Ann. Med. Health Sci. Res., vol. 4, no. 6, pp. 928-32, Nov. 2014.

[25] Sehhati F. et al. "Effect of Continuous midwifery care on length of Labor," J. Caring Sci., vol. 1, no. 1, pp. 47-52, May 2012.

[26] Maro EW. et al. "Ten years trend in maternal mortality at Kilimanjaro Christian Medical Center Tanzania, 2003-2012: A descriptive retrospective tertiary hospital based study," Asian Pacific J. Reprod., vol. 5, no. 3, pp. 214-220, May 2016.

[27] Krüger C. “Essay 3 Maternal mortality in Tanzania," 2007

\section{Figures}




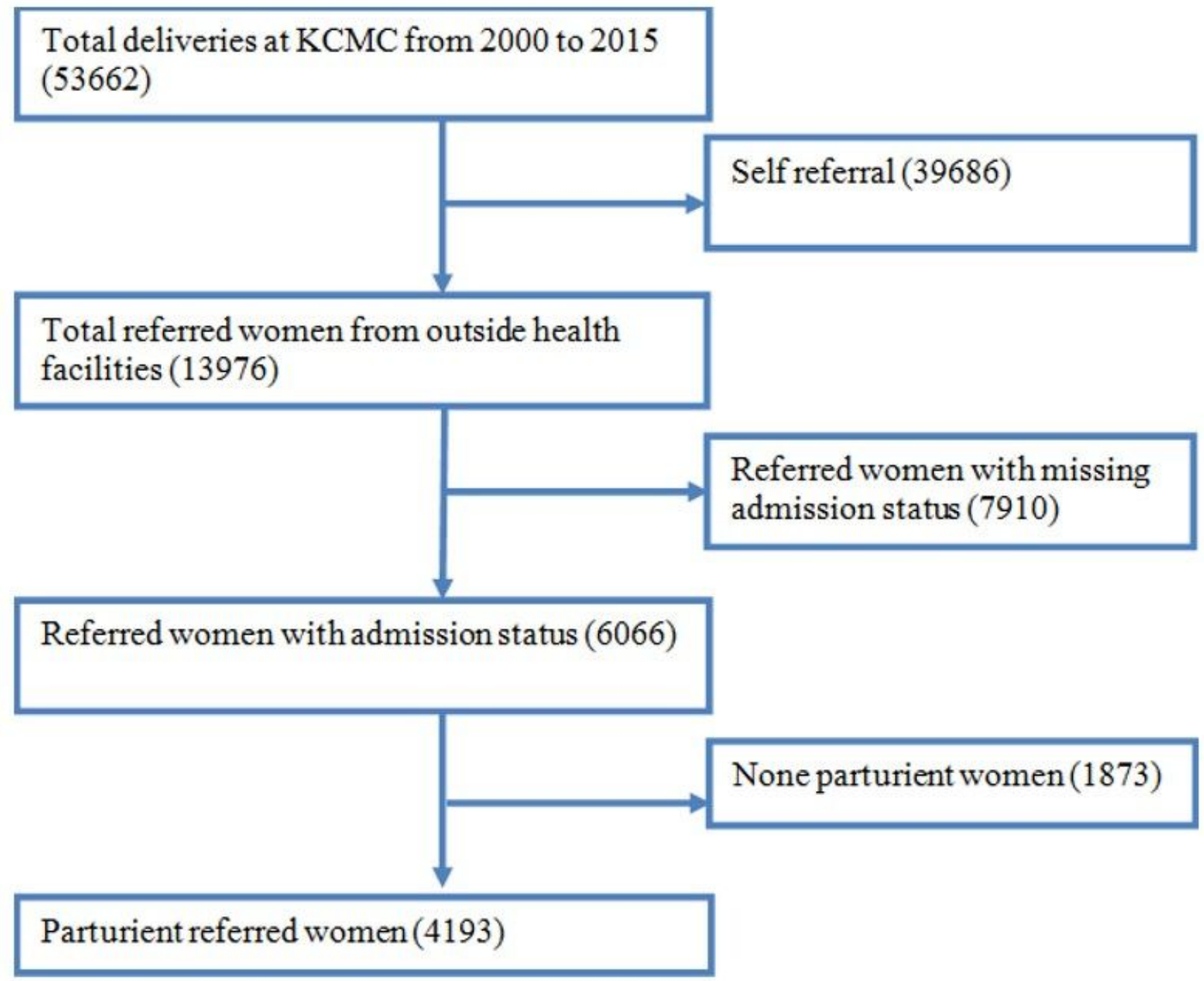

Figure 1

Flow chart of pregnant women delivered at KCMC and those excluded and included in the study. 